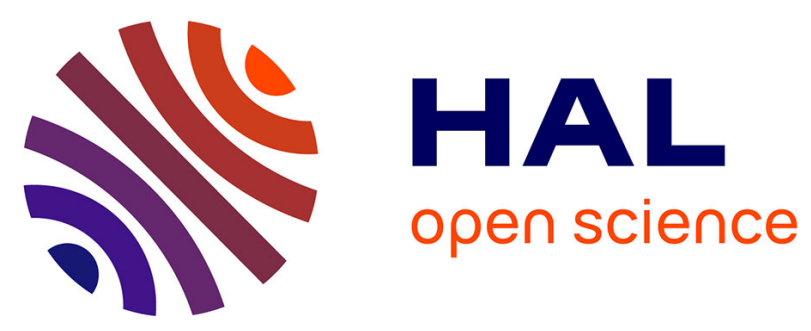

\title{
Analysis of grasping strategies and function in hemiparetic patients using an instrumented object
}

Nathanael Jarrasse, Markus Kuhne, Nick Roach, Asif Hussain, Sivakumar

Balasubramanian, Etienne Burdet, Agnès Roby-Brami

\section{- To cite this version:}

Nathanael Jarrasse, Markus Kuhne, Nick Roach, Asif Hussain, Sivakumar Balasubramanian, et al.. Analysis of grasping strategies and function in hemiparetic patients using an instrumented object. 13th International Conference on Rehabilitation Robotics (ICORR), 2013, Seattle, United States. 10.1109/ICORR.2013.6650379 . hal-03161259

\section{HAL Id: hal-03161259 \\ https://hal.science/hal-03161259}

Submitted on 5 Mar 2021

HAL is a multi-disciplinary open access archive for the deposit and dissemination of scientific research documents, whether they are published or not. The documents may come from teaching and research institutions in France or abroad, or from public or private research centers.
L'archive ouverte pluridisciplinaire HAL, est destinée au dépôt et à la diffusion de documents scientifiques de niveau recherche, publiés ou non, émanant des établissements d'enseignement et de recherche français ou étrangers, des laboratoires publics ou privés. 


\title{
Analysis of grasping strategies and function in hemiparetic patients using an instrumented object
}

\author{
Nathanaël Jarrassé*, Markus Kühne*, Nick Roach ${ }^{\dagger}$, Asif Hussain ${ }^{\dagger}$, Sivakumar Balasubramanian $^{\ddagger}$, \\ Etienne Burdet ${ }^{\dagger}$ and Agnès Roby-Brami* \\ * Institute of Intelligent Systems and Robotics, University Pierre et Marie Curie, CNRS - UMR 7222, Paris, France \\ Email:\{jarrasse, roby-brami\}@isir.upmc.fr \\ $\dagger$ Department of Bioengineering, Imperial College of Science, Technology and Medicine, London SW7 2AZ, UK \\ Email:\{n.roach, asif.hussain09, e.burdet\}@imperial.ac.uk \\ \# Tecnalia, Parque Cientìfico y Tecnologico de Bizkaia - C/ Geldo. Edificio 700. E-48160 Derio (Bizkaia), Spain
}

\begin{abstract}
This paper validates a novel instrumented object, the iBox, dedicated to the analysis of grasping and manipulation. This instrumented box can be grasped and manipulated, is fitted with an Inertial Measurement Unit (IMU) and can sense the force applied on each side and transmits measured force, acceleration and orientation data wirelessly in real time. The iBox also provides simple access to data for analysing human motor control features such as the coordination between grasping and lifting forces and complex manipulation patterns. A set of grasping and manipulation experiments was conducted with 6 hemiparetic patients and 5 healthy control subjects. Measures made of the forces, kinematics and dynamics are developed, which can be used to analyse grasping and contribute to assessment in patients. Quantitative measurements provided by the iBox reveal numerous characteristics of the grasping strategies and function in patients: variations in the completion time, changes in the force distribution on the object and grasping force levels, difficulties to adjust the level of applied forces to the task and to maintain it, along with movement smoothness decrease and pathological tremor.
\end{abstract}

\section{INTRODUCTION}

The impairment of grasping ability is very frequent in stroke survivors: more than half of them remain with an impairment of the upper extremity largely contributing to the disability in the daily life function [1] and to the limitation of social participation. However, despite the importance of this question and the large number of rehabilitation techniques recently proposed for the rehabilitation of reaching to grasp and grasping [2], there still lacks comprehensive studies on grasping function and manipulation after stroke. In addition, there is also a lack of pertinent methods for the assesment of grasping function in hemiparetic patients. Most of the existing methods are either based on the measure of elementary impairments (e.g. pinch force measured by a dynamometer) or on functional tests quantified by rough scores summarizing the success/failure of the attempts to grasp a battery of objects [3]. The existing methods do not allow analyzing precisely neither the determinants of complex impairments induced by stroke nor the mechanisms of their consequences on function. In particular, they miss the ability of the patients to develop alternative grasping strategies if the precision grip is impaired [4].

We have developed an instrumented object in order to bridge the gap between elementary measures and functional scores for a better therapy and follow-up of stroke patients. The first practical aim was to afford quantitative measures able to be applied to a large range of upper-extremity function, i.e. from the detection of slight impairments to the evaluation of gross grasping disability. The second aim was that the method should be usable in clinics, and thus easy to use, robust and low-cost. In addition, our aim was to provide a generic tool to analyze hand function in healthy subjects. This article first describes the technical solution used for the mechanical design and instrumentation of this device. Then, preliminary results obtained during experiments with healthy subjects and brain-injured patients are presented in order to put forward some pertinent variables that could be used for fundamental investigation about grasping and, looking further ahead, for clinical assessment.

\section{STATE OF THE ART FOR THE QUANTITATIVE ANALYSIS OF PREHENSION}

\section{A. Precision grip}

In healthy subjects, many existing studies are devoted to precision grip. The precision grip as defined by Napier in his pioneering study [5] is a multipulpar opposition between fingers and thumb for precision tasks. However experimental studies were most often focused on the precision grip between the thumb and index, analysed thanks to an instrumented object measuring load force and grip force coordination during a lifting task. Extensive studies have largely demonstrated that grip force was tightly coupled to load force and that both grip and load force rates were both modulated in an anticipatory way as a function of object properties (size, shape, contact surface) [6], [7]. The maximum grip force is produced in an anticipatory way in order to remain slightly above the margin of security to avoid object slip. In addition, the regulation of grip force during the holding of the object is strongly dependant of tactile afferents from the skin of the finger pulp [8]. Some studies [9], [10] showed that the dynamics of object displacement could also be anticipated as observed on the evolution of the grip force signal.

\section{B. Complex hand-object interactions}

More complex hand-object interactions have been studied with specific research-oriented instrumentation. The extensive studies of tri-digit [11] or multifinger [12] dynamic coordination use specific instrumented objects that needed placing 
precisely each finger on a force sensor. These studies demonstrated a sharing pattern of forces between fingers, based on anatomical constraints, and finely regulated synergies in order to generate forces and moments on the object as a function of task instruction. However, these methods are not usable in patients who cannot precisely position the tip of their fingers on sensors. Other studies on the coordination of hand and fingers for grasping an object recorded the multiple joint rotations during reaching and grasping thanks to an instrumented glove or to optic methods [13]. These analyses showed that the complex kinematic of hand and fingers could be explained by additive synergies combined as a function of the object shape and anticipated during the reaching phase. These complex methods are not used in clinical practise.

\section{Hemiparetic patients}

There are several but relatively few analysis of grasping function in hemiparetic patients in grasping [14] or a grip to lift task [15]. They show a longer time before lift-off, impairments of grip-lift coordination with excessive grasping forces. The analysis of power grasp is generally limited to the measure of force with a Jamar Dynamometer. Nowak and Hermsdorfer [16] developed an autonomous instrumented object which has been use to document grasping in several pathological conditions. However this instrumented object was limited to 2 forces measurement: the grip force and the vertical load force. Presently, there is no device that can be used in clinics to detect more complex manipulation difficulties, nor methods that can be used for the discrimination and analysis of alternative strategies using the whole hand for grasping.

\section{METHODS}

\section{A. Instrumented object}

The apparatus used in this study is an addition to the existing set of instrumented objects for rehabilitation and assessments presented previously in [17]. It has been developed by the Human Robotics Group of Imperial College London. It comes in the form of a parallelepiped (see Fig. 1) whose dimensions are $108 \mathrm{~mm}$ length, $70 \mathrm{~mm}$ width and $40 \mathrm{~mm}$ depth and whose weight is 340 grams.

The object uses an embedded electronic board MIMATE (Multimodal Interactive Motor Assessment and Training Environment, see [18] for more informations). This object measures its accelerations, rotational velocities and orientations (thanks to an IMU) along with the value of distinct forces applied normally to its six surfaces (up to $20 \mathrm{~N}$ ). It then transmits these values wirelessly through bluetooth to a distant computer, at a $100 \mathrm{~Hz}$ frequency approximatively.

\section{B. Subjects}

Five healthy subjects (22-28 years old, 2 women) volunteered to participate to this preliminary study, as well as six hemiparetic patients (34-62 years old, 4 women) from the department of Physical Medicine and rehabilitation of the Raymond Poincaré Hospital in Garches, France. The patient's clinical and functional data are summarised in Table I. Functional activity of patients was evaluated by the Frenchay Arm Test (FAT) [19]. This tests gives results from 0 to 5 since it scores five prototypal gestures as possible (1) or not (0). All subjects were naive to the system and experiments.

\section{Installation and tasks}

The participants seated in front of a table on which the object was put in front of his/her midline. Seven tasks were studied (see Fig. 1), with three repetitions per task. The first five tasks were grip-lift tasks: the instruction was to lift the object a few centimeters above the table to hold it a few second and to put it back on the table in the following conditions.
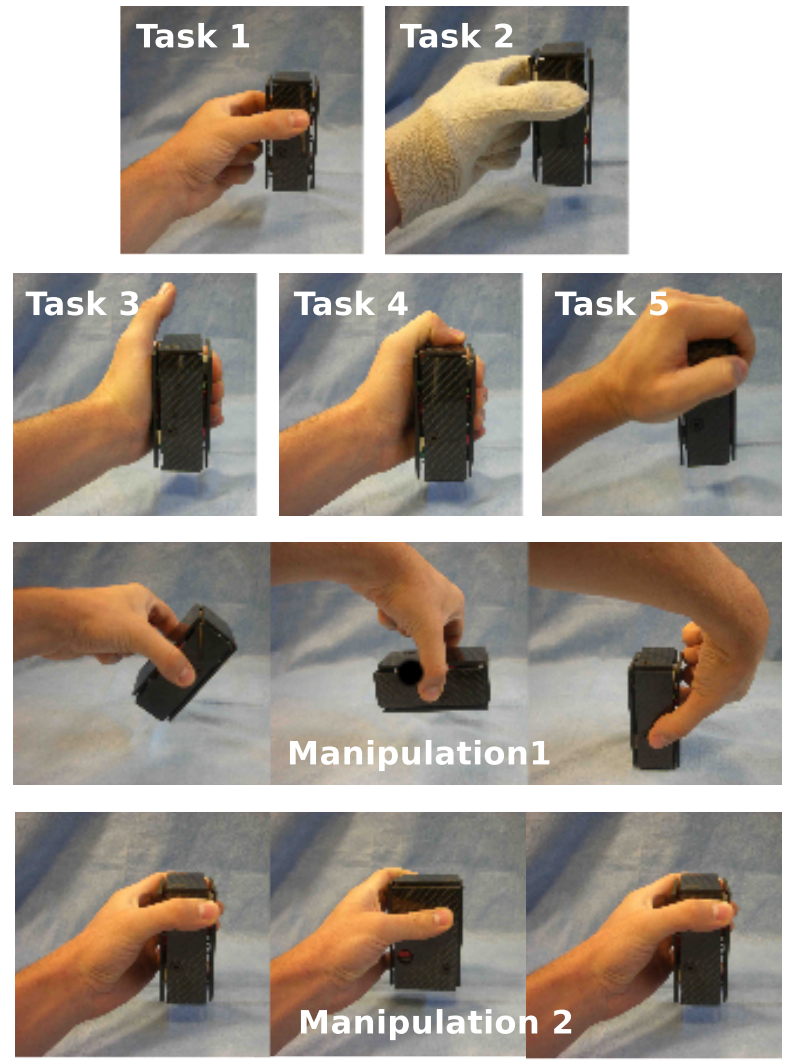

Fig. 1. Illustrations of the 5 grasping tasks and the 2 manipulation tasks.

- Task 1, Precision grip: along the lateral sides of the object (arrows on figure).

- $\quad$ Task 2, Slippery precision grip: same as previous, but with a cotton glove.

- $\quad$ Task 3, Whole hand grasping without thumb: between the palm and the four fingers around the object.

- Task 4, Whole hand grasping with thumb: same as previous, but with the thumb on the top side.

- $\quad$ Task 5, Global grasping from the top of the object.

The two last tasks were more complex manipulation tasks.

- Manipulation 1, Tilting: the instruction was to lift the object and to put it back on the table its top below (rotation around an horizontal axis). The instruction did not precise if the object could slide between the fingers.

- Manipulation 2, Handling : the instruction was to lift the object and to rotate it around its main axis with the fingers. 
TABLE I. CLINICAL CHARACTERISTICS OF PATIENTS

\begin{tabular}{|c|c|c|c|c|c|}
\hline ID & Cause & Pathology & FAT & Exp. performed & Remarks \\
\hline 1 & Stroke & Mild left hemiparesis & 3 & Task 1-7, Manip. 1-2 & Manip.2 unsuccessful \\
\hline 2 & Stroke & Moderate right hemiparesis, aphasia & 4 & Task 1-2 & Technical problem \\
\hline 3 & Stroke & Mild right hemiparesis & 4 & Task 1-5, Manip. 1-2 & Thumb use for task 3. Manip.2 unsuccessful \\
\hline 4 & Traumatic brain injury & Severe paresis, hand orthopedic condition & 0 & Task 1-3 & Grasp between thumb and forearm \\
\hline 5 & Stroke & Mild right hemiparesis, spasticity, hypoesthesia & 3 & Task 1,3-5, Manip. 1-2 & Manip 2 unsuccessful \\
\hline 6 & Angiome at age 7 , Mid brain Stroke & Spasticity, syncinesia, cerebellar syndrom & 5 & Task 1-5, Manip. 1-2 & - \\
\hline
\end{tabular}

\section{Analysis of the results}

The present analysis is limited to the force measured by the load-cells and kinematics and dynamics data computed thanks to the embedded IMU. These data were interpolated according to the time stamp to obtain vectors with $0.001 \mathrm{~s}$ time samples $(100 \mathrm{~Hz})$. The first step was to detect the times of interest by an automatic analysis visually checked (Fig. 2). The onset $\left(t_{r}\right)$ and end $\left(t_{f}\right)$ of grasping were determined with a threshold of $0.2 \mathrm{~N}$ for the sum of the 6 forces. The force sensor of the bottom indicates the loading/unloading of the object on the table. The object is lift-off when the value is $-3.4 \mathrm{~N}$ (offset weight of the object). The timing was measured as the time when the bottom force reached (lifting $t_{l}$ ) or quitted (deposit $t_{d}$ ) the plateau, with a threshold of $0.1 \mathrm{~N}$. The time of the peak force $\left(t_{p}\right)$ was measured between $t_{l}$ and the plateau and the values of force data at this time noted. Positive force peaks measured by the bottom sensor were also detected and evaluated. The means of the three repetitions were computed, as well as the means over the different subjects of the experiment.

\section{ANALYSIS OF GRASPING COORDINATION IN HEALTHY SUBJECTS}

\section{A. Grip-lift tasks}

1) Precision grip: Figure 2 shows typical example of lift with a precision grip. During precision grip, the force increased

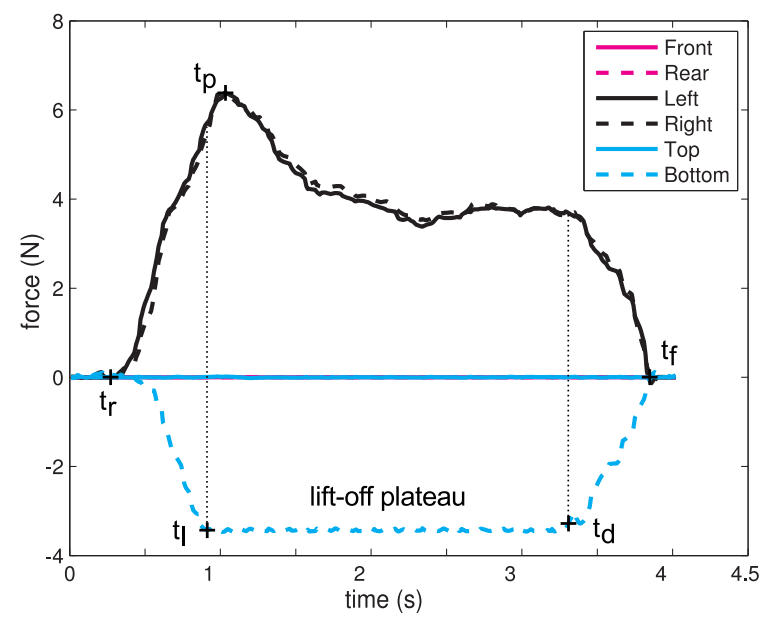

Fig. 2. Example of force data during precision grip (task 1). Time scale $10 \mathrm{~ms}$. Times: onset of movement $t_{r}$, lifting $\left(t_{l}\right)$, peak force $\left(t_{p}\right)$ deposit $\left(t_{d}\right)$ and end of movement $\left(t_{f}\right)$. Each trace is one trial. The force sensors are indicated in legend.

regularly on both lateral sensors until lift at $t_{l}(492 \pm 48 \mathrm{~ms}$ after $t_{r}$ ). The mean lateral forces were respectively $7.9 \pm 0.9$ and $7.2 \pm 0.9$ at $t_{l}$ and continued to increase to reach $8 \pm$ 0.96 and $7.8 \pm 0.9$ at $t_{p}\left(637 \pm 63 \mathrm{~ms}\right.$ after $\left.t_{r}\right)$. In parallel the force on the bottom sensor decreased. After lift-off the force on the lateral sensors decreased to reach a plateau, (sometimes followed by a later and smaller peak) until the time of deposit $t_{d}$, when they were $6.5 \pm 1$ and $6.14 \pm 0.9$. Then, the force of the lateral sensor decreased and that of the bottom sensor increased during $442 \pm 57 \mathrm{~ms}$ until $t_{f}$. The precision grip task with a glove gave a similar picture with much higher levels of force (reached $15.10 \pm 1.06$ and $14.86 \pm 1.05$ ) that remained high during the whole lift-off period. The timing of lifting was slower $\left(t_{l}\right.$ was attained $652 \pm 98 \mathrm{~ms}$ and $t_{p} 866 \pm 127 \mathrm{~ms}$ after $t_{r}$, i.e. around $200 \mathrm{~ms}$ more than without glove) but the deposit was performed faster (in $280 \pm 47 \mathrm{~ms}$ ).

2) Whole hand grasping: During the task with whole hand grasping, the evolution followed a similar picture, except that the force increased also in a greater number of sensors and reached a greater level. Figure 3 shows typical example of lift with a power grasp. The timing of lifting with the whole hand

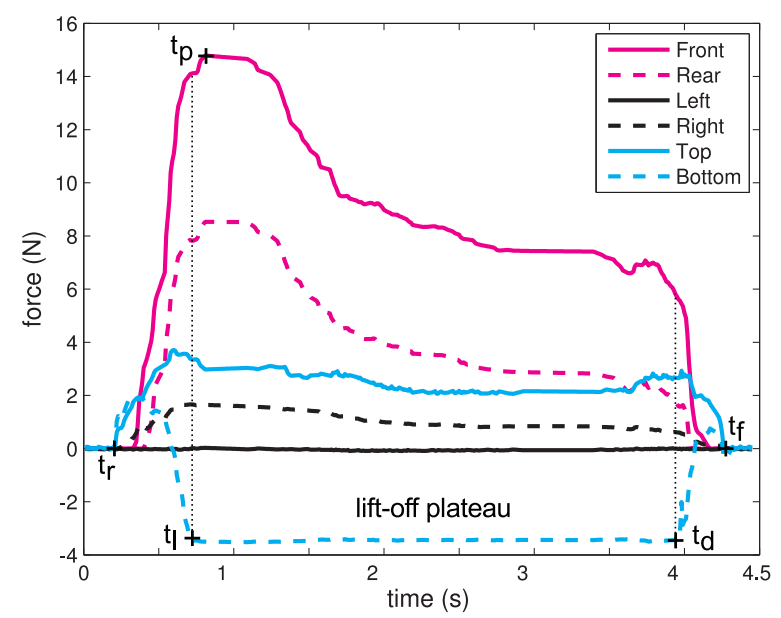

Fig. 3. Example of force data during power grasp with thumb (task 5 ) in a representative healthy subject. The force sensors are indicated in legend.

(task 3) was similar to that of precision grip ( $t_{l}$ was attained $407 \pm 54 \mathrm{~ms}$ and $t_{p} 647 \pm 149 \mathrm{~ms}$ after $t_{r}$ ). In contrast, the timing for the tasks 4 and 5 was markedly slower $\left(t_{l}\right.$ was attainted in respectively $872 \pm 87 \mathrm{~ms}$ and $802 \pm 108 \mathrm{~ms}$ and $t_{p}$ in respectively $1118 \pm 154 \mathrm{~ms}$ and $930 \pm 115 \mathrm{~ms}$ ). The whole hand grasping tasks were also characterized by a positive peak on the bottom sensor that we called 'bump' indicating that the participant pressed downward on the object. For task 3, this bump was short $(120 \pm 28 \mathrm{~ms})$ small $(0.7 \pm 0.1)$ and inconstant. In contrast, it was greater and longer for tasks 4 and 5 (respectively $2.6 \pm 0.2$ and $2.2 \pm 0.3$ attained at $325 \pm$ $45 \mathrm{~ms}$ and $287.3 \pm 51 \mathrm{~ms}$ after $t_{r}$ ).

3) Grasp-lift coordination: The co-variation between the bottom force signal indicating the loading/unloading of the 

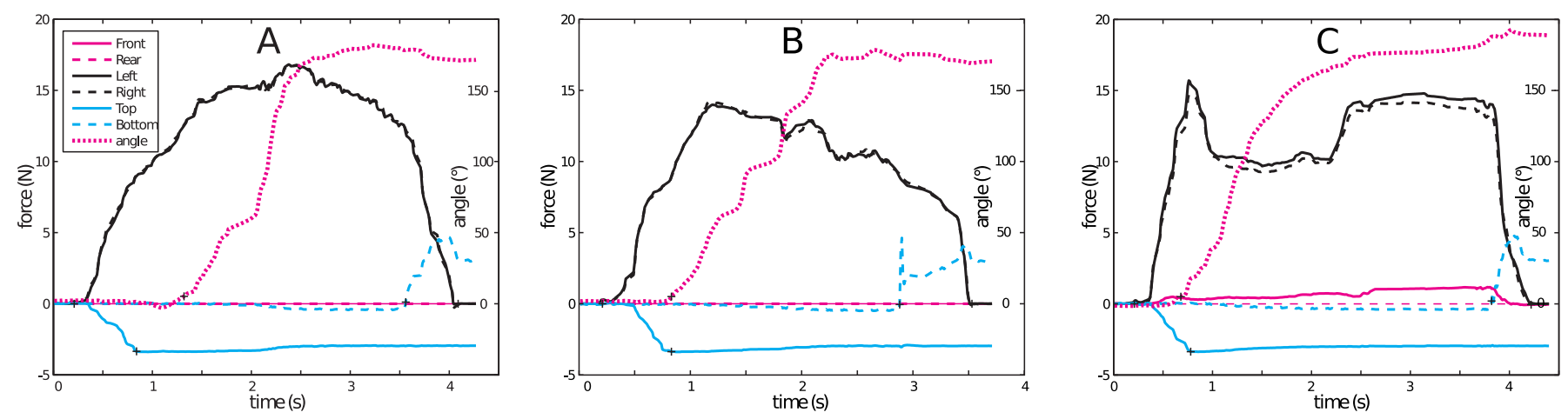

Fig. 4. Examples of three strategies for the tilting task in healthy subjects: Fig. 4.A shows tilting by hand rotation with constant grasping force, Fig. 4.B rotation through pushing on the rear face and relaxation of grasping force, and finally Fig. 4.C shows tilting started before lifting strategy. Same legend as Figure 2.

object on the table and the grasping forces was studied during lift-off (between $t_{r}$ and $t_{l}$ ) and deposit (between $t_{d}$ and $t_{f}$ ).

Figure 5 shows the results in a representative subject. The diagrams shows that, during precision grip, the grasping force for lifting (left) is tightly related to the loading of the object (negative values on the force bottom sensor). This is also observed for the deposit period (right). Precision grip performed with the glove gave similar results with higher levels of force. In contrast, grasping with the whole hand was more irregular: the grasping force increase while the object is pushed on the table before lifting (indicated by positive values on the force bottom sensor).
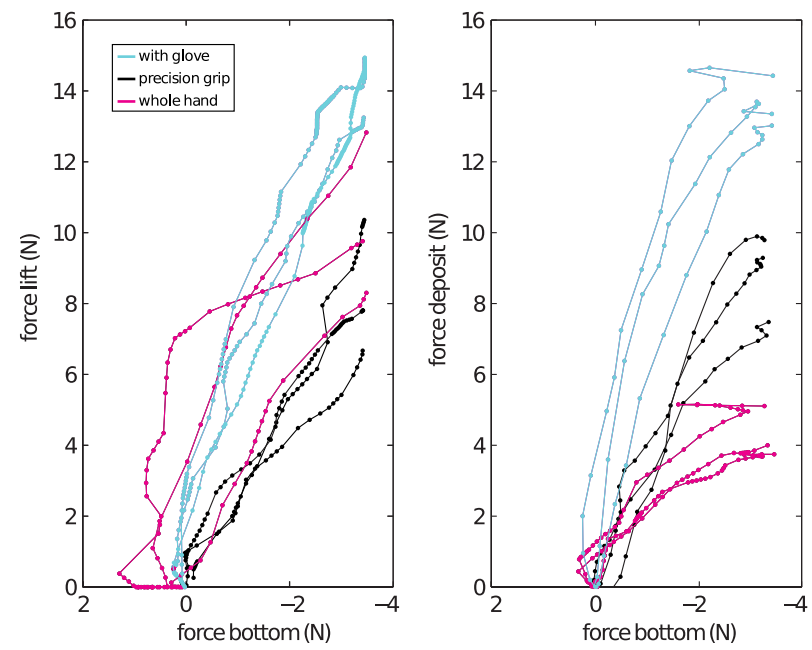

Fig. 5. Coordination of grasping and bottom forces during lifting between $t_{r}$ and $t_{l}$ (left) and deposit between $t_{d}$ and $t_{f}$ (right) (see Fig. 2 for time events placement). The diagram shows the value of the left sensor for precision grip and of the front sensor for whole hand grasping as a function of the value of the bottom sensor. Each line represents one repetition in one representative subject (3).

\section{B. Manipulation tasks}

1) Tilting: During the tilting task, several strategies were observed. In some cases, the participant tilted the object by rotating the hand, without relaxing his grasping strength (Fig. 4A). In contrast, the tilt was obtained by pushing on the rear face, inducing a brisk rotation while relaxing the grasping strength (e.g. in Fig. 4C where the tilt begins even before lifting). In other intermediate cases (Fig. 4B), the object was tilted in several steps each accompanied by a decrease of the grasping strength.

2) Handling: The task to turn the object around its main axis imposes complex sequential movement of the fingers. According to the constraint of this task, all the subjects made the rotation in several steps. Figure 6 shows a representative example, each rotation step illustrated by a grey zone.

After lifting the object, the first rotation step (A) was obtained by passing the grasping strength from the lateral to the front sensors. The second step (B) was made without change of the main contact face of the box. The main rotation occurred in (C) with rapid alternation of force contact on the lateral and front-rear faces. After the last rotation, the object is returned to its original orientation and grasping pattern then released on the table. Note that during all transitions, the object is maintained by the bottom face (the offset value of the sensor is reduced). A similar picture was obtained in most of the trials.

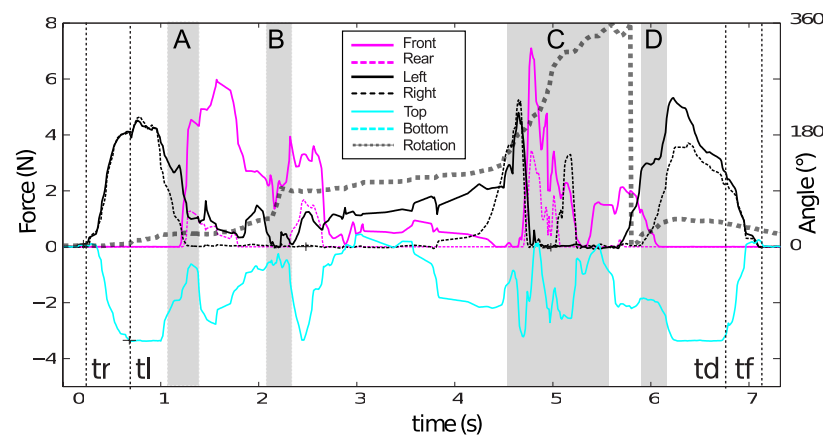

Fig. 6. Example of handling task in healthy subjects. Same legend as Figure 2.

\section{DIVERSITY OF GRASPING IMPAIRMENT AND STRATEGIES OBSERVED IN HEMIPARETIC PATIENTS.}

We present here some preliminary and simple analysis of data gathered with 4 brain-injured hemiparetic patients from 35 to 62 years old (excluding subject 4 who had also hand 
orthopedic problems, and subject 2 who was only able to complete task 1 and 2 because of some technical problems). Our goal here is to define a certain number of indexes or dependant variables that could qualify patient recovery state and assesses his/her performances.

\section{A. Grasping forces analysis}

Figure 7 shows that the sequence of events for precision grip (task 1) in a patient with a moderate disorder is similar to that observed in healthy subjects. However, there are several important differences: the onset of lifting is slow and irregular with a "bump" on the object characterized by an increase of the force signal on the bottom sensor. The force signal from the lateral grasped sides increase irregularly before attaining the peak force, then do not exhibit a regular plateau as in healthy subjects but rather a sharp and irregular decrease with oscillations. The deposit is irregular with a vibration.

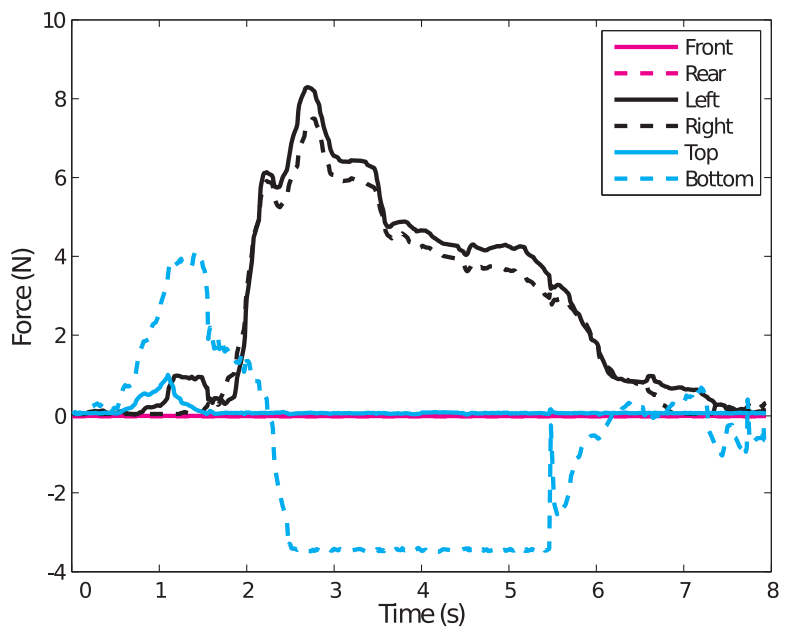

Fig. 7. Example of force data during precision grip performed by an hemiparetic patient.

1) Grasping force levels and variations: We first quantified the amount of grasping force required to lift the object. We considered the data from Tasks 1 and 2 (precision grip with and without a glove) and 3 (power grasping without the use of the thumb) and made some comparison between results obtained with the population of healthy subjects and 4 poststroke hemiparetic chronic patients.

For this purpose we therefore only considered forces applied on the the functional faces of the object during lift-off. Mean force levels and standard deviations are shown in the Figure 8. We observe that the average grasping force level is higher with patients than with healthy subjects, with an average increase of $47.18 \%$ for tasks 1 and 3 . Results from task 2 (with the glove) have to be considered carefully because of the load-cell saturation that occurred around $17 \mathrm{~N}$ : one can imagine that similar value of force increase would have been observed if the range of the load-cells was larger, instead of the presently measured $9.6 \%$ of increase. The force STD values that are increasing by $177 \%, 49.5 \%$ and $36 \%$ in tasks 1,2 and 3 respectively may illustrate the large variations of the level of grasping force applied by patients (important decrease) during lift-off.

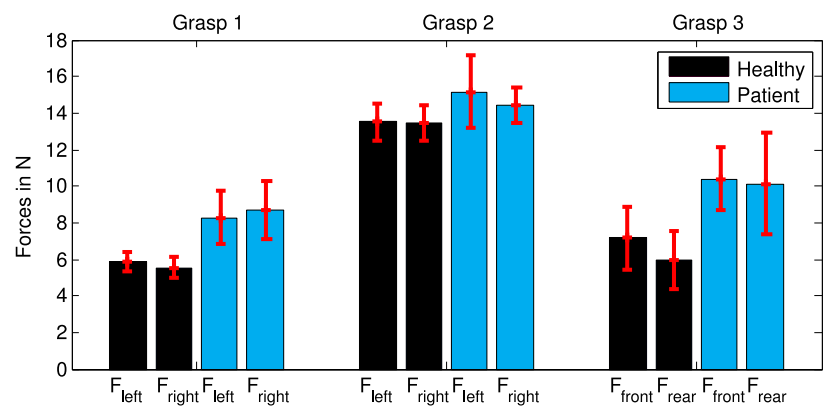

Fig. 8. Grasping force level (and standard deviation) on functional surfaces during tasks 1,2 (precision grip with and without glove) and 3 (power grip without thumb) calculated for when the object is lifted up and averaged over 5 healthy subjects and 4 patients, and the 3 trials.

2) Grasping force repartition: In order to study the alteration of the grasping strategies of hemiparetic patients, rather than problems of local force generation or control, we used a "radar' representation of the means of force applied on each of the six surfaces of the iBox during lift-off. Grasping strategy can indeed be seen as the repartition of forces among the different surfaces of the object, i.e. the shape of the hexagon on a radar plot, whereas the scaling of this hexagon would give informations on force level variations. As an example, Figure 9 shows the force repartition for task 5 (grasping from above) for healthy and patients.
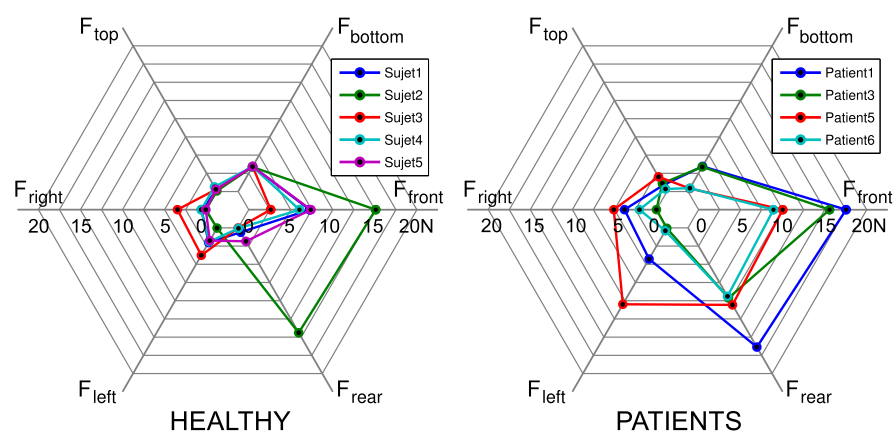

Fig. 9. Mean force repartition (averaged during the time when the object is lifted) for task 5 (grasping from above) for each subject and patient (averaged over the 3 trials).

Apart subject 2, healthy subjects seem to use similar grasping strategies with some scaling variations (force level variations). In comparison, patients appear to use very different force repartition to complete the task. This is particularly easy to spot with task 5 (grasping from above) that could be performed with different force repartitions, contrary to other experiments (like precision grip or power grasp) in which strategy variability is more constrained by the task instructions.

\section{B. Grasping dynamics}

1) Grasping force oscillations: An interesting characteristic of patient sensorimotor pathology can also be extracted from the grasping force profile: oscillations can be observed in the force application that seems to be correlated with some position oscillations of the grasped and held object (measured through the vertical accelerations of the object). A typical 
example (patient 6 performing task 1, precision grip) can be seen in Figure 10, in which one $2 \mathrm{~Hz}$ oscillation frequency can be identified on the amplitude spectrum of the acceleration obtained with a simple Fast Fourier Transform (FFT).
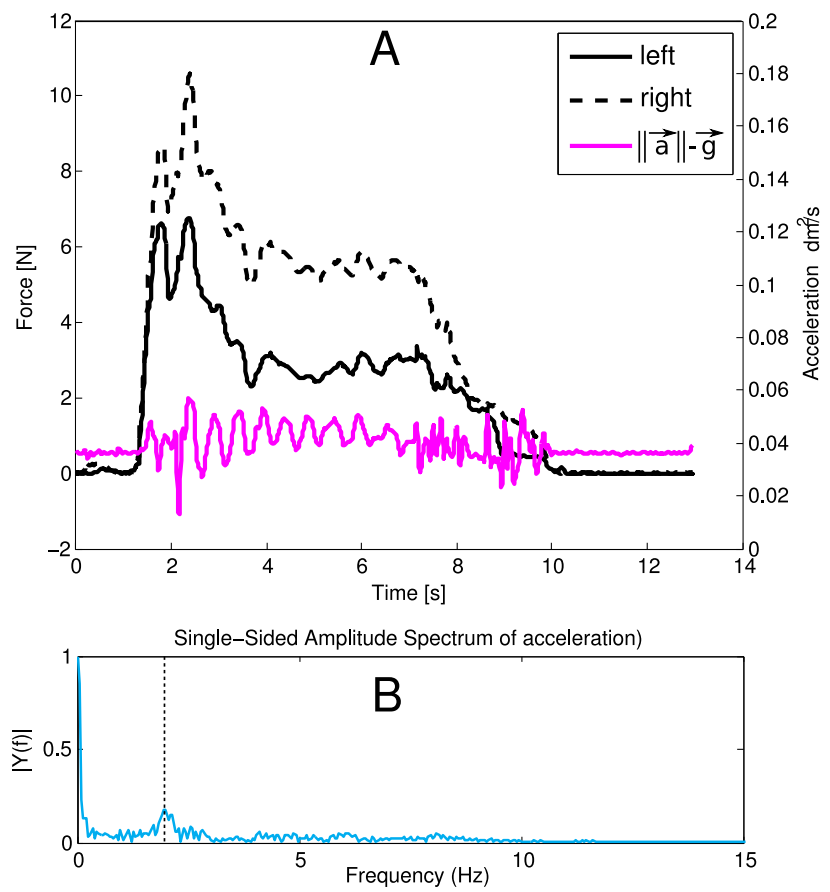

Fig. 10. Task 1 (precision grip) performed by a patient: A. Grasping forces and norm of the acceleration of the object. B. Amplitude spectrum of the acceleration during the task.

2) Smoothness of the movement: The Spectral Arc Length (SAL) smoothness metric developed in [20] was applied to angular velocity of the subtasks in each trial. SAL is a robust, dimensionless measure of the length, and thus the complexity, of a frequency spectrum curve over the bandwidth of interest. For this study, the upper frequency bound was set to $20 \mathrm{~Hz}$ as this is typically sufficient to capture normal and abnormal human motions [20]. Fig. 11 presents the smoothness value of

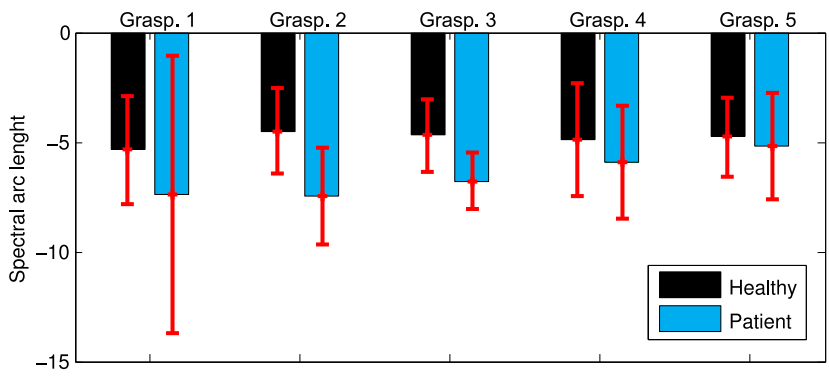

Fig. 11. Measure of smoothness (spectral arc length) averaged over subjects (4 patients and 5 healthy subjects) and trials (3) for the grasping and lifting tasks (task 1-5)

the vertical velocities during tasks 1 to 5 . Angular velocities were calculated by numerical differentiation on the vertical acceleration, to which was subtracted the gravity acceleration, thanks to the reconstruction of the object posture made from the information from the 9 DoF IMU.
A clear decrease of the smoothness can be observed with patients, ranging from $9 \%$ (task 5) up to $65 \%$ (task 2).

\section{Manipulation tasks}

1) Tilting task: Four patients succeeded in performing the tilting task (manipulation 1).

Figure 12 illustrates two examples. In Fig. 12.A, after an initial the patient turns the object with several partial releases of the grasping strength, exhibiting a strategy similar to that of healthy subject illustrated Fig. 4.B. The patient illustrated in Fig. 12.B is more irregular. Tilting is performed after a first phase of tilting in the opposite direction and the repartition of force exceeds the two lateral sensors.
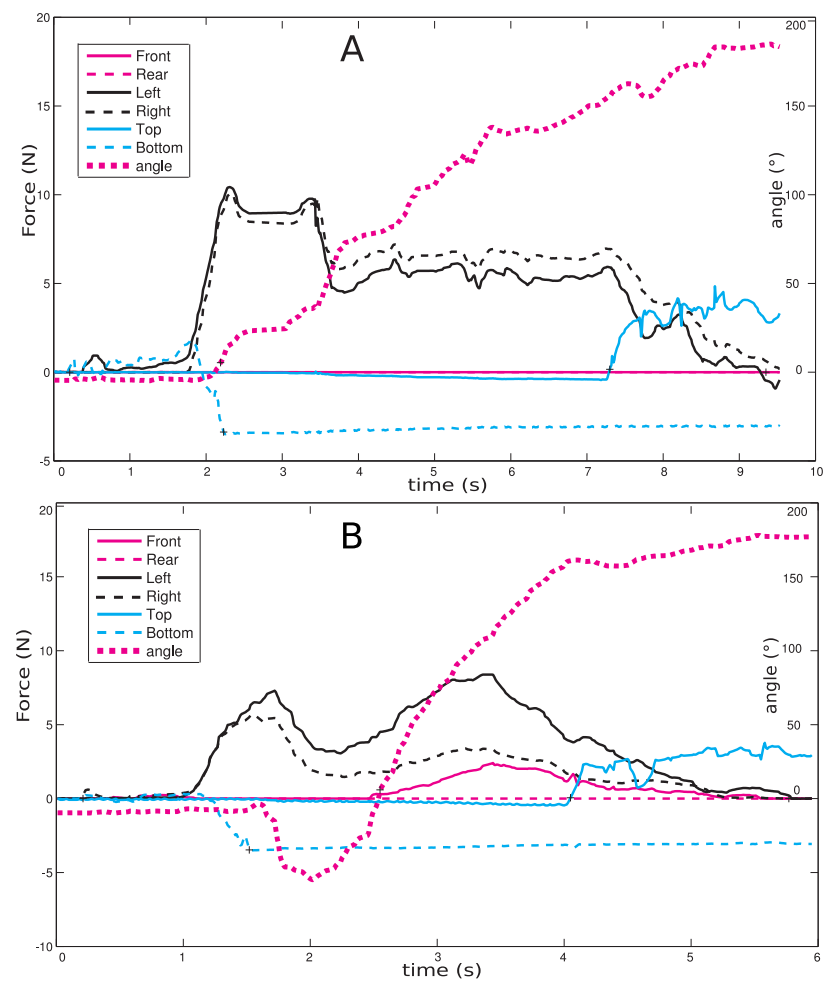

Fig. 12. Two examples of three strategies for the tilting task in healthy subjects: Fig. 12.A shows a strategy with several partial releases whereas Fig. 12.B is similar to healthy subject strategy. Same legend as Figure 2.

2) Handling task: Despite repeated attempts, most of the patients did not succeed in the handling task (manipulation 2). This shows that they were not able to make the individuated finger movements needed to turn the object within the hand. Only one patient succeeded (patient 6), but she did not use the 5th finger to support the weight of the object in a vertical position. Rather, she held the object more horizontally in such a way that its weight was mostly supported by the palm.

\section{DISCUSSION}

The preliminary results obtained with the iBox open several tracks for further research. Even if it does not provide the positioning and the kinematics of each finger over the surfaces, it gives access to reliable force, kinematics and dynamics 
variables and can enable experimenters to finely characterise grasping strategies in healthy subjects and in physically or neurologically affected individuals.

The grasp-lift coordination of precision grip which has been largely studied previously is important since it highlights the anticipatory control of grip force as a function of object properties before lifting. We show here that the data from the iBox can be used to further study this anticipatory control. Moreover, the iBox gives access to additional quantitative (force and motion) data during more complex hand-object interaction such as grasping with or without the thumb and other manipulation tasks. Unimanual manipulation of an object is a particularly complex and difficult task since the coordinated fingers must simultaneously move the object and ensure its equilibrium. Although these tasks typically demonstrate human dexterity and are the topic of many recent studies in humanoid and anthropomorphic robotics (see e.g.[21]), they have paradoxically been little studied quantitatively in humans [12].

In this paper we examined two different manipulation tasks. We observed that for tilting task the healthy subjects chose three different strategies, probably differing according to usual motor habits, avoiding or not uncomfortable hand postures [22] at the risk of object slipping, or to different understandings of the task. This illustrates the great variability of human strategies during object/tool manipulation. The handling task was still more demanding but all the healthy subjects succeeded. The force data highlighted interesting phases of force production on different sides of the object alternating with the holding of the object by the bottom face, presumably thanks to the fifth finger. These strategies obviously need further studies to be characterised and studied in various sensorimotor or environmental contexts.

We also demonstrated in this paper that the Box device can be used for the physiopathological analysis of motor performance in patients suffering from sensorimotor disorders. All the patients succeeded in the grip to lift tasks, even the most impaired with associated orthopaedic problems. The presented data show that the patients trying to make precision grips exhibit several atypical features relative to healthy subjects: they apply excessive grasping force, push downward before grasping, the grasp to lift phase is longer and more irregular with eventually some slips, the distribution of force is spread over more than two opposite sides, the time evolution of the force signal is irregular with sharp decrease or irregularities and oscillations, and motion smoothness is altered.

The results of patients trying to grasp with precision grip are roughly similar to the whole-hand grasping performed by healthy subjects (tasks 3-5). Further studies are needed with quantitative comparison in order to precise whether grasping in hemiparetic patients can be explained by impairments of precision grip, replaced by whole hand grasping, or if both precision grip and whole hand grasping are really impaired. The application of excessive forces (more than is required to complete the lifting tasks) may correspond to two different problems: a motor control problem with which the patient is not able to finely tune his force production; and a sensory problem, with which he/she is not able to perceive the amount of force applied, and regulate it thanks to a feedback loop. Observed sharp decrease of force after the first peak is con- sistent with a loss of perception that could bring patients to apply more force than is required and then, thanks to visual feedback (object is not slipping), to minimise the grasping force. According to these preliminary results we also recently updated the design of the iBox, in order to measure grasping forces up to $20 \mathrm{~N}$.

The periodic irregularities during the holding of the object may have several physiopathological causes such as tremor and spasticity, which can be inferred from a frequency analysis [23]. In the patient with mid-brain stroke whose data are shown in Fig. 10, the $2 \mathrm{~Hz}$ frequency suggests a cerebellar tremor rather than spasticity as initially suspected (clonus has a higher frequency around $7 \mathrm{~Hz}$ ). Furthermore, numerous information can be extracted from the repartition of the force on the sensors embedded in the Box object. The grasping strategies can be be characterised through the analysis of the force repartition among the object faces beyond a generic analysis of the force levels measured by load cells. Accordingly, future experiments may let the patient grasp the object as they spontaneously prefer. Presumably, this would lead to a variety of hand-object force repartition that could be categorised by an automated pattern analysis. This kind of analysis could be also used to quantify the transitions between complex object manipulation phases in healthy and impaired subjects.

Finally, the iBox seems to be suitable for the clinical assessment of grasping, and the various tasks proposed with the iBox may encompass a variety of functional levels. Patients with a severe motor impairment can take the object with gross whole hand grasping while patients with a mild disorder may exhibit specific impairments when performing complex manipulation tasks. The case studies of a few subjects presented in this paper illustrated the capabilities of iBox to characterise sensorimotor impairments and enabled us to develop suitable metrics, which may complement assessment using standard clinical scales. Nevertheless, some more rigorous experimental campaigns with larger populations of patients and subjects (also with similar age range) along with statistical analysis will now be conducted in order to establish proper comparisons between sensorimotor strategies.

\section{ACKNOWLEDGMENT}

We acknowledge Pr. Bussel and the team of occupational therapists from the department of neurological rehabilitation, Hôpital Raymond Poincaré, for their constructive suggestions and assistance for the experiments with the patients.

This work has been partly supported by UK-UKIERI IND/CONT/E/1172/182, the FP7-PEOPLE-2012-ITN project No 317488: CONTEST and by a Short Term Scientific Mission funding from the COST Action TD1006 European Network on Robotics for NeuroRehabilitation.

\section{REFERENCES}

[1] H. S. Jorgensen, H. Nakayama, H. O. Raaschou, J. Vive-Larsen, M. Stoier, and T. S. Olsen. Outcome and time course of recovery in stroke. part i: Outcome. the copenhagen stroke study. Arch Phys Med Rehabil, 76(5):399-405, 1995.

[2] J. W. Krakauer. Motor learning: its relevance to stroke recovery and neurorehabilitation. current opinion in Neurology, pages 84-90, 2006. 
[3] S. L. Wolf, P. A. Catlin, M. Ellis, A. L. Archer, B. Morgan, and A. Piacentino. Assessing motor function test as outcome measure for research in patients after stroke. Stroke, 32(7):1635-9, 2001.

[4] D. Bensmail, J. Robertson, C. Fermanian, and A. Roby-Brami. Botulinum toxin to treat upper-limb spasticity in hemiparetic patients: grasp strategies and kinematics of reach-to-grasp movements. Neurorehabil Neural Repair, 24(2):141-51, 2010.

[5] J. R. Napier. The prehensile movements of the human hand. The Journal of bone and joint surgery. British volume., 38-B(4):902-13, 1956.

[6] R.S. Johansson and G. Westling. Coordinated isometric muscle commands adequately and erroneously programmed for the weight during lifting task with precision grip. Exp Brain Res, 71:59-71, 1988.

[7] R.J. Flanagan, K Merritt, and R. Johansson. predictive mechanisms and object representations used in object manipulation. In D. Nowak and J. Hermsdoerfer, editors, Sensorimotor control of grasping, physiology and pathophysiology, pages 161-177. Cambridge University Press, Cambridge, 2009.

[8] R. S. Johansson and K. J. Cole. Sensory-motor coordination during grasping and manipulative actions. Curr Opin Neurobiol, 2(6):815-23, 1992.

[9] J. R. Flanagan and A. M. Wing. Modulation of grip force with load force during point-to-point arm movements. Exp Brain Res, 95(1):13143, 1993.

[10] J. Hermsdorfer, E. Hagl, and D. A. Nowak. Deficits of anticipatory grip force control after damage to peripheral and central sensorimotor systems. Hum Mov Sci, 23(5):643-62, 2004.

[11] S. A. Winges, S. E. Eonta, J. F. Soechting, and M. Flanders. Multidigit control of contact forces during rotation of a hand-held object. $J$ Neurophysiol, 99(4):1846-56, 2008.

[12] V. M. Zatsiorsky and M. L. Latash. Multifinger prehension: an overview. J Mot Behav, 40(5):446-76, 2008.

[13] M Santello, M Flanders, and J F Soechting. Postural hand synergies for tool use. The Journal of Neuroscience, 18(23):10105-10115, 1998.

[14] P. G. Lindberg, N. Roche, J. Robertson, A. Roby-Brami, B. Bussel, and M. A. Maier. Affected and unaffected quantitative aspects of grip force control in hemiparetic patients after stroke. Brain Res, 1452:96-107, 2012.

[15] D. A. Nowak, J. Hermsdorfer, and H. Topka. Deficits of predictive grip force control during object manipulation in acute stroke. J Neurol, 250(7):850-60, 2003.

[16] D. A. Nowak and J. Hermsdorfer. Objective evaluation of manual performance deficits in neurological movement disorders. Brain Res Rev, 51(1):108-24, 2006.

[17] A. Hussain, N. Roach, S. Balasubramanian, and E. Burdet. A modular sensor-based system for the rehabilitation and assessment of manipulation. In Haptics Symposium (HAPTICS), 2012 IEEE, pages $247-254$, march 2012.

[18] N. Roach, A. Hussain, and E. Burdet. Learning to design rehabilitation devices through the h-card course: Project-based learning of rehabilitation technology design. Pulse, IEEE, 3(6):51 -58, nov. 2012.

[19] Derick T Wade. Measurement in neurological rehabilitation. Oxford University Press, USA, 1992.

[20] S. Balasubramanian, A. Melendez-Calderon, and E. Burdet. A robust and sensitive metric for quantifying movement smoothness. Biomedical Engineering, IEEE Transactions on, 59(8):2126 -2136, aug. 2012.

[21] A. Bicchi. Hands for dexterous manipulation and robust grasping: A difficult road toward simplicity. Robotics and Automation, IEEE Transactions on, 16(6):652-662, 2000.

[22] D. A. Rosenbaum, K. M. Chapman, M. Weigelt, D. J. Weiss, and R. van der Wel. Cognition, action, and object manipulation. Psychol Bull, 138(5):924-46, 2012.

[23] A. Machowska-Majchrzak, K. Pierzchala, and S. Pietraszek. Analysis of selected parameters of tremor recorded by a biaxial accelerometer in patients with parkinsonian tremor, essential tremor and cerebellar tremor. Neurol Neurochir Pol, 41(3):241-50, 2007. 